\title{
Caracterización de la comunidad de aves asociada a los humedales de zonas bajas del departamento del Tolima, Colombia
}

\author{
Characterization of the bird community associated with the lowland wetlands of \\ Tolima, Colombia
}

\author{
Gustavo F. Pacheco-Vargas, Jessica N. Sánchez-Guzmán y Sergio Losada-Prado
}

\section{Resumen}

En Colombia los estudios sobre la avifauna en humedales interiores son escasos. En el caso del departamento del Tolima no se cuenta con información detallada, por lo cual se requieren trabajos que provean datos sobre este tópico. Por tal motivo, este estudio tiene como objetivo caracterizar la comunidad de aves asociada a los humedales de zonas bajas del departamento. Para esto, se estudiaron 13 humedales ubicados por debajo de 1000 m s.n.m. Se registraron 147 especies, distribuidas en 44 familias y 18 órdenes. Los humedales con mayor número de especies fueron Toqui Toqui (78) y El Edén (65), mientras que Chicoalí (22), El Samán y El Gavilán (27, cada uno) registraron el menor número; sin embargo, no se encontraron diferencias significativas en la composición de la avifauna de los humedales evaluados. Predominaron las especies de las categorías ecológicas para aves II (35 \%) y III (38 \%) y en menor medida fueron registradas las especies pertenecientes a la categoría Ia (1\%). A pesar de la alta intervención a la que están sometidos los humedales del bosque seco tropical en el Tolima, estos ecosistemas aún sustentan un número importante de especies.

Palabras clave. Avifauna. Bosque seco tropical. Categorías ecológicas. Cuenca del río Magdalena. Intervención antrópica. Paisaje agropecuario.

\begin{abstract}
In Colombia, studies on wetland birds are scarce. Specifically in the department of Tolima, there is no detailed information. Therefore, studies on this topic are required. In this sense, this study aims to characterize the community of birds associated with lowland wetlands in Tolima. Thirteen wetlands located below $1000 \mathrm{~m}$ a.s.l. were studied, and 147 species of birds, distributed in 44 families and 18 orders, were found. The wetlands with the highest number of species were Toqui Toqui (78) and El Edén (65), while Chicoalí (22), El Samán and El Gavilán (27 species each) had the lowest number of species. However, no significant differences were found in the composition of the avifauna for the evaluated wetlands. Ecological categories II (35\%) and III (38\%) prevailed and, to a lesser extent, species belonging to category Ia $(1 \%)$ were recorded. Despite the high intervention to which the tropical dry forest wetlands in Tolima are subject to, these ecosystems still support a significant number of species.
\end{abstract}

Keywords. Agricultural landscape. Anthropic intervention. Birds. Ecological categories. Magdalena River basin. Tropical dry forest. 


\section{Introducción}

El término humedales hace referencia a una gran variedad de ambientes interiores y costeros los cuales tienen como característica principal el papel del agua en la estructura del ecosistema (Blanco, 1999). Estos entornos son esenciales en la regulación del ciclo hídrico, la retención de sedimentos, el control de erosión, la estabilización microclimática, la regulación de los ciclos de nutrientes y la descomposición de biomasa terrestre; además, son base de la productividad de los sistemas acuáticos, la estabilidad e integridad de los ecosistemas y la retención de dióxido de carbono (Naranjo et al., 1998).

Entre los grupos faunísticos más diversos en los humedales se encuentran las aves (Weller, 1994), de las cuales algunas familias hacen uso permanente de sus recursos a tal punto que han desarrollado diversas adaptaciones morfológicas que les permiten aprovecharlos eficientemente (p. ej. Anatidae, Ardeidae, Threskiornithidae, Charadriidae, entre otros), mientras otras únicamente utilizan estos ambientes temporalmente en el periodo de nidificación y cría (Passeriformes) (Blanco, 1999). Un aspecto que resalta la importancia de las aves dentro de los humedales es que pueden ser consideradas como bioindicadores de su estado con base en las siguientes evidencias: 1 . Se han detectado declives en sus poblaciones a causa de la desecación o degradación de los humedales (Delany et al., 1999); 2. El tamaño, la forma, la profundidad, la presencia y la distribución de la vegetación influye sobre la composición y abundancia de las aves acuáticas presentes (Velazquez, 1992; Craig y Beal, 1992; Safran et al., 1997); 3. La cercanía entre humedales favorece la diversidad y abundancia de las aves (Brown y Dinsmore, 1986). Por lo anterior, este grupo taxonómico es fundamental en la identificación, caracterización y establecimiento de límites de los humedales según criterios biológicos y ecológicos (Parra, 2014).

Pese a la importancia de estos ecosistemas en Colombia, los estudios relacionados con los humedales interiores son escasos (Castillo y Puyana, 2004; Estela y López-Victoria, 2005; Johnston-González et al., 2006; Álvarez-León, 2009; Lara, 2011; Gutiérrez et al., 2014), por lo que es necesario adelantar investigaciones para conocer su biodiversidad y de este modo proponer medidas de conservación (Lasso et al., 2014). El objetivo de este estudio fue caracterizar la comunidad de aves asociada a los humedales de zonas bajas del departamento del Tolima.

\section{Materiales y métodos}

Área de estudio. Este estudio se llevó a cabo en 13 humedales ubicados por debajo de 1000 m s.n.m. en el bosque seco tropical de la región del valle alto del río Magdalena en el departamento del Tolima (Figura 1, Figura 2, Anexo 1). El bosque seco tropical se define como aquella formación vegetal que presenta una cobertura boscosa continua y se distribuye entre los 0 a $1000 \mathrm{~m}$ s.n.m. (Pizano y García, 2014), se caracteriza por presentar una evapotranspiración potencial que supera a la precipitación, una temperatura anual promedio igual o mayor a $25^{\circ} \mathrm{C}$, y una precipitación anual entre 700 a 2000 mm (Sánchez-Azofeifa et al., 2005).

Registro de aves. La compilación de especies se realizó teniendo en cuenta las listas publicadas por Cruz et al. (2010) para los humedales Río Viejo, La Coya, Saldañita, el Edén, Toqui Toqui, Losada-Prado y Pacheco-Vargas (2015a, 2015b, 2015c, 2015d, 2015e) para Albania, Azuceno, El Samán, La Huaca y Las Garzas, y LosadaPrado et al. (2016a, 2016b, 2016c) para Chicoalí, El Gavilán y Caracolí. Estos regis-tros se obtuvieron mediante muestreos rápidos en cada humedal de la siguiente forma: 1 . Puntos de conteo cada $100 \mathrm{~m}$, con una duración de 10 minutos por punto referenciado, se realizaron 6 puntos, para un total de 60 minutos de observación por día. 2. Se instalaron $72 \mathrm{~m}$ de redes de niebla las cuales permanecieron abiertas entre las 06:0010:00, para un total de $24 \mathrm{~h}$ red/día. Cada humedal se visitó una vez entre los años 2010 a 2015. 


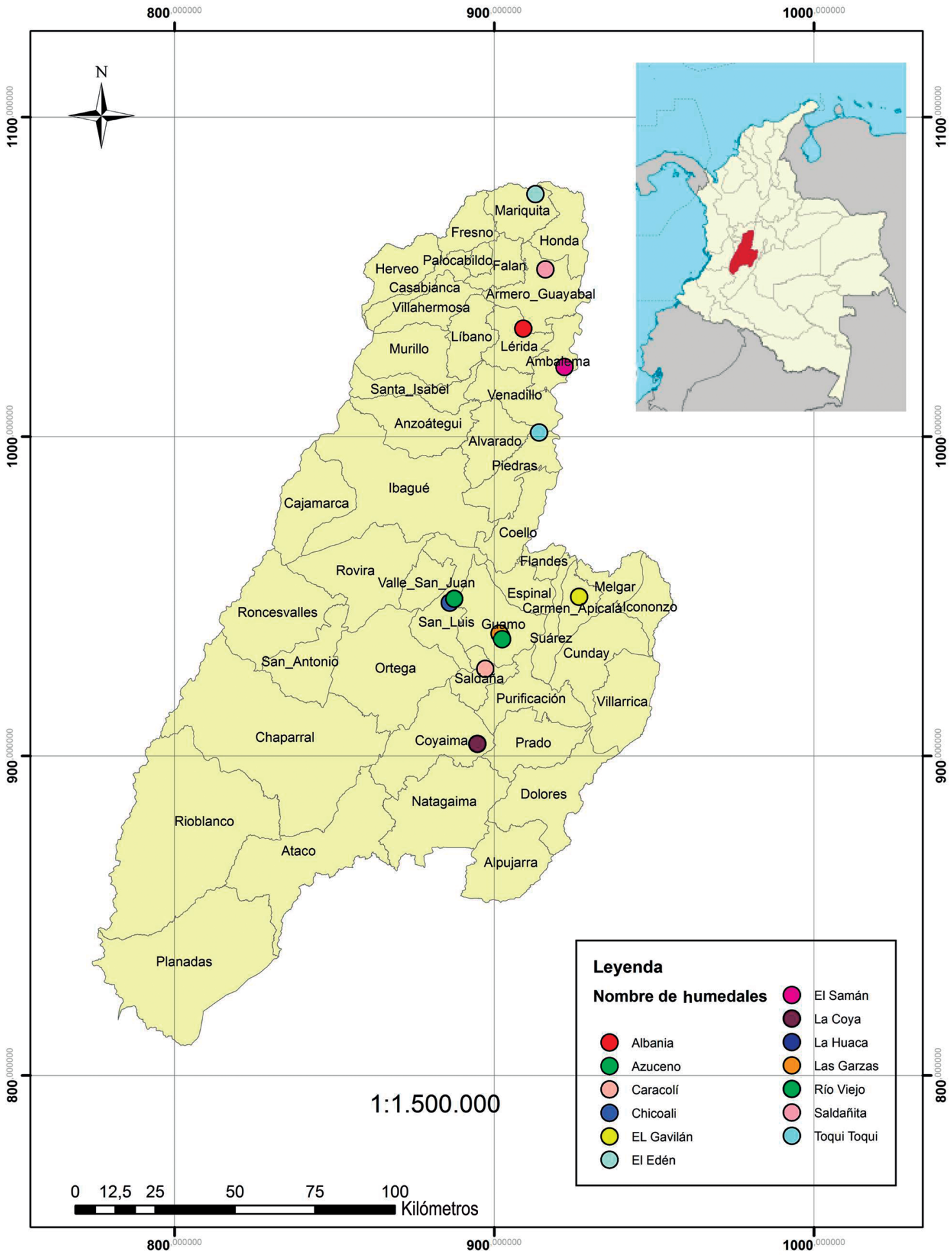

Figura 1. Mapa de ubicación de los humedales de tierras bajas muestreados en el bosque seco tropical del departamento del Tolima. 

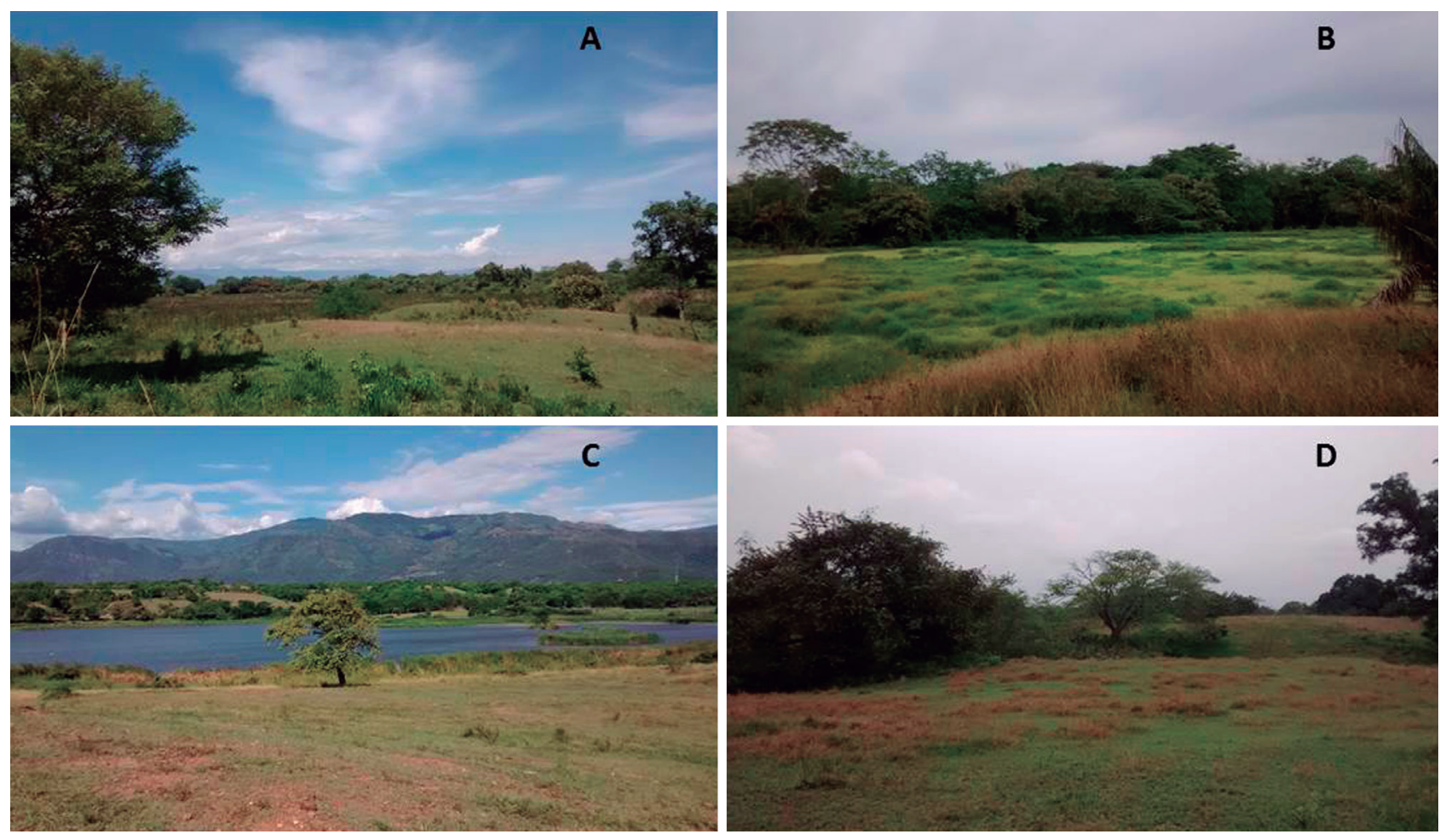

Figura 2. Fotografías de cuatro de los humedales de tierras bajas muestreados en el bosque seco tropical del departamento del Tolima. A) Humedal La Huaca, B) humedal Las Garzas, C) humedal El Samán y D) humedal Azuceno.

El listado final de especies sigue la secuencia recomendada por la Unión Americana de Ornitólogos (Remsen et al., 2017), y se tuvo en cuenta el listado de aves endémicas, casiendémicas y especies de interés de ChaparroHerrera et al. (2013). Para verificar las aves migratorias registradas se siguió a Naranjo et al. (2012). Además, se corroboró si las especies registradas se encuentran en alguna categoría de amenaza según la Unión Internacional para la Conservación de la Naturaleza (UICN) y si están incluidas en algún apéndice de la Convención sobre el Comercio Internacional de Especies Amenazadas de Fauna y Flora Silvestres (CITES).

Análisis de información. La representatividad del muestreo se evaluó estimando el número de especies esperadas por medio de una curva de acumulación de especies, empleando los estimadores Chao 1 y ACE. Se tuvo en cuenta el número de especies representados por uno o dos individuos (singletos y doubletons, respectivamente) mediante el programa EstimateS 9.0 (Colwell, 2013).

Se realizó un análisis de categorías ecológicas para las especies registradas según Stiles y Bohórquez (2000). Dichas categorías fueron propuestas para aves exclusivamente y son: I. Especies de bosque: a. Especies restringidas al bosque primario o poco alterado. b. Especies no restringidas al bosque primario o poco alterado. II. Especies de bosque secundario o bordes de bosque, o de amplia tolerancia. III. Especies de áreas abiertas. IV. Especies acuáticas: a. Especies asociadas a cuerpos de agua sombreadas o con la vegetación densa al borde del agua, evitando áreas abiertas o soleadas. b. Especies asociadas a cuerpos de agua sin sombra, orillas abiertas o con vegetación baja. V. Especies aéreas: a. Especies que requieren por lo menos parches de bosque. b. Especies indiferentes a la presencia de bosque o que prefieren áreas más 
abiertas. La asignación de las categorías ecológicas a las especies registradas se realizó de acuerdo con los análisis de Losada-Prado y Molina-Martínez (2011).

La diversidad beta fue evaluada teniendo en cuenta el índice de Bray-Curtis, el cual se representó por medio de un dendrograma con ayuda del programa PAST 3.0 (Hammer et al., 2001). Además, se realizó un análisis de escalamiento multidimensional (NMDS) teniendo en cuenta la abundancia de especies por humedal y las siguientes variables no métricas: 1 . Ubicación del humedal (rural o urbano) y 2. Tipo de cobertura alrededor del humedal (potrero, cultivo, bosque). Se conformaron tres grupos: I. Azuceno, Caracolí, El Gavilán, Las Garzas (urbano, potrero). II. La Coya, Chicoalí, Toqui Toqui (rural, potrero). III. Albania, El Samán, La Huaca, Río Viejo (predominan extensiones de cultivo o bosque). Para la realización de este análisis se utilizó el programa PRIMER 6 (PRIMER-E, 2006). Por último, usando los mismos agrupamientos generados en el análisis NMDS, se efectuó un análisis de similaridad no paramétrica (ANOSIM) para detectar diferencias en la composición de la avifauna de los humedales usando PAST 3.0 (Hammer et al., 2001). En los análisis anteriormente descritos no se tuvieron en cuenta las siguientes especies debido a que presentan una gran movilidad y se consideró que no dependen principalmente del humedal para su desarrollo: Cathartidae: Cathartes aura, Coragyps atratus; Accipitridae: Elanus leucurus, Buteogallus meridionalis, Rupornis magnirostris; Apodidae: Streptoprocne zonaris; Falconidae: Herpetotheres cachinnans, Caracara cheriway, Milvago chimachima, Falco femoralis; Psittacidae: Amazona ochrocephala, Psittacara wagleri; Tyrannidae: Tyrannus savana; Turdidae: Catharus ustulatus; Parulidae: Parkesia noveboracensis, Setophaga petechia.

\section{Resultados}

Riqueza de especies. Se registraron 147 especies de aves, distribuidas en 44 familias y 18 órdenes (Figura 3). La familia más numerosa fue
Tyrannidae (26 especies), seguida de Thraupidae (13 especies) y Ardeidae (8 especies). Se registraron 2 especies endémicas (Myiarchus apicalis y Euphonia concinna), 7 especies casi-endémicas y Phaethornis anthophilus como especie de interés (especies que tienen entre el 40-49\% de su distribución en Colombia según ChaparroHerrera et al., 2013), 7 especies migratorias, Psittacara wagleri como especie casi-amenazada (IUCN), Dendrocygna autumnalis dentro del apéndice III de la CITES y 20 especies dentro del apéndice II de la CITES. El humedal con mayor número de especies fue Toqui Toqui (78), seguido por El Edén (65), y Río Viejo y Caracolí (45). El menor número de especies se registró en la Laguna Chicoalí (22), El Samán y Gavilán (27) (Anexo 2).

Representatividad del muestreo. Según los estimadores ACE y Chao1, la representatividad general del muestreo fue del 93-94\%. Las curvas de acumulación muestran un aumento al completar el máximo número de localidades muestreadas. Por otra parte, las curvas de especies raras presentan una leve tendencia al descenso (singletons y doubletons) (Figura 4).

Categorías ecológicas. Predominan especies de las categorías ecológicas II (35 \%) y III (38 \%); enseguida se encuentra la categoría IVb (14\%). En menor medida se encuentran presentes las especies de la categoría Ia (1 \%) (Figura 5).

Diversidad beta. El análisis de similaridad de Bray-Curtis definió tres grupos. El primero de ellos conformado por los humedales Caracolí, El Samán, Las Garzas y La Coya; el segundo por El Edén, Saldañita, La Huaca, Azuceno, El Gavilán y Albania; el tercero por Chicoalí y Río Viejo; el humedal con menor similitud fue Toqui Toqui (Figura 6). El NMDS es congruente con los resultados del análisis de similaridad (Figura 7). Sin embargo, no se encontraron diferencias significativas en la composición de la avifauna de los grupos establecidos (ANOSIM, R=0,07; $p=0,23)$. 

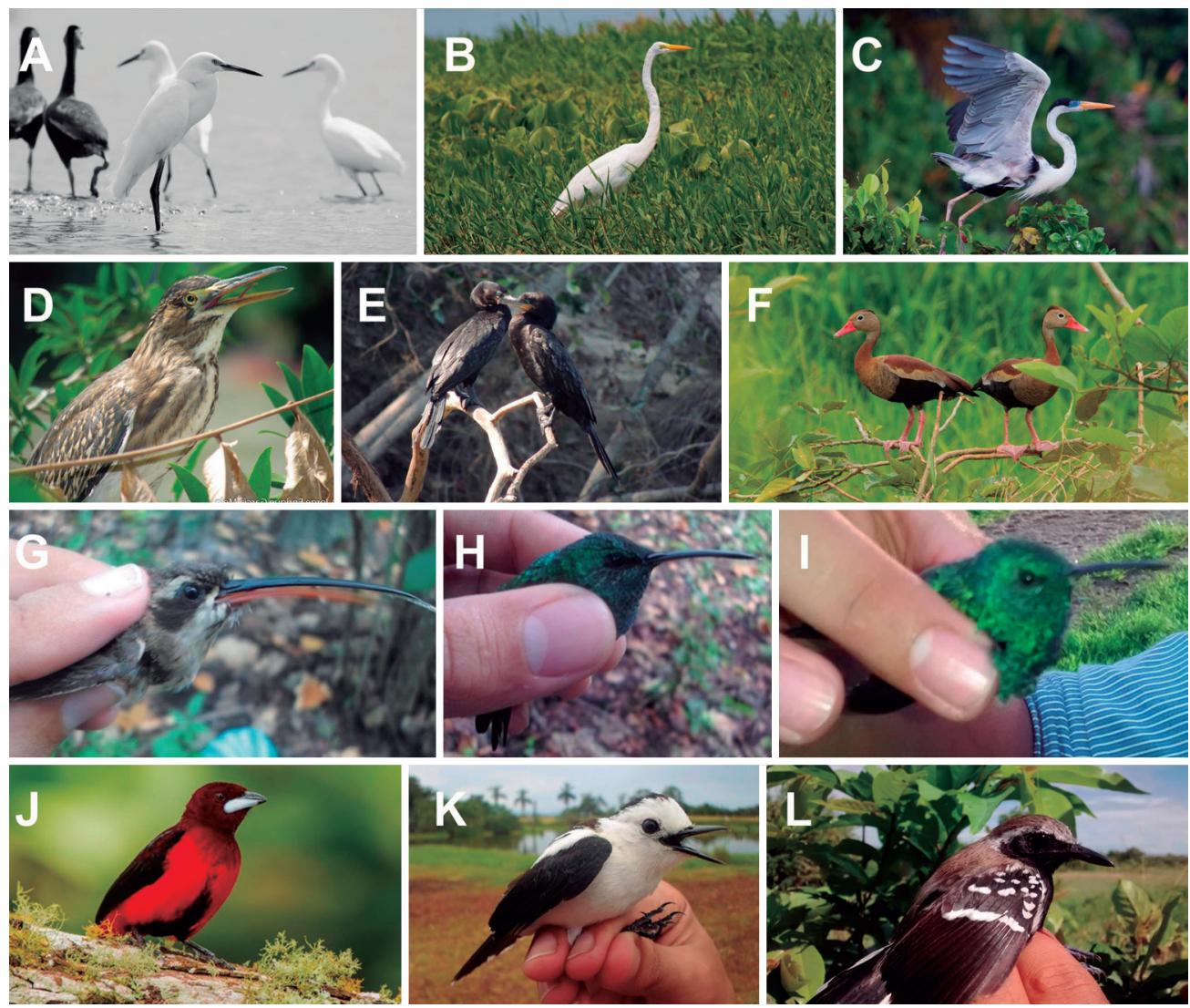

Figura 3. Algunas especies representativas de los humedales de tierras bajas del bosque seco tropical del departamento del Tolima. A) Egretta thula, B) Ardea alba, C) Ardea cocoi, D) Nycticorax nycticorax, E) Phalacrocorax brasilianus, F) Dendrocygna autumnalis, G) Phaethornis anthophilus, H) Chlorostilbon gibsoni, I) Lepidopyga goudoti, J) Ramphocelus dimidiatus, K) Fluvicola pica y L) Formicivora grisea.

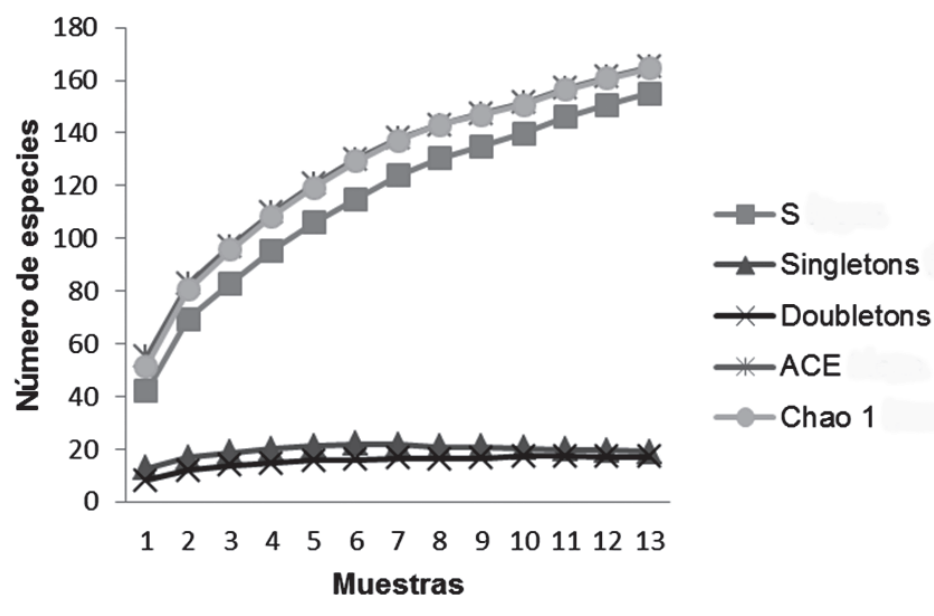

Figura 4. Curva de acumulación de especies de aves registradas en los humedales de tierras bajas $(<1000 \mathrm{~m}$ s.n.m.) del bosque seco tropical del departamento del Tolima durante los años 2010-2015. 


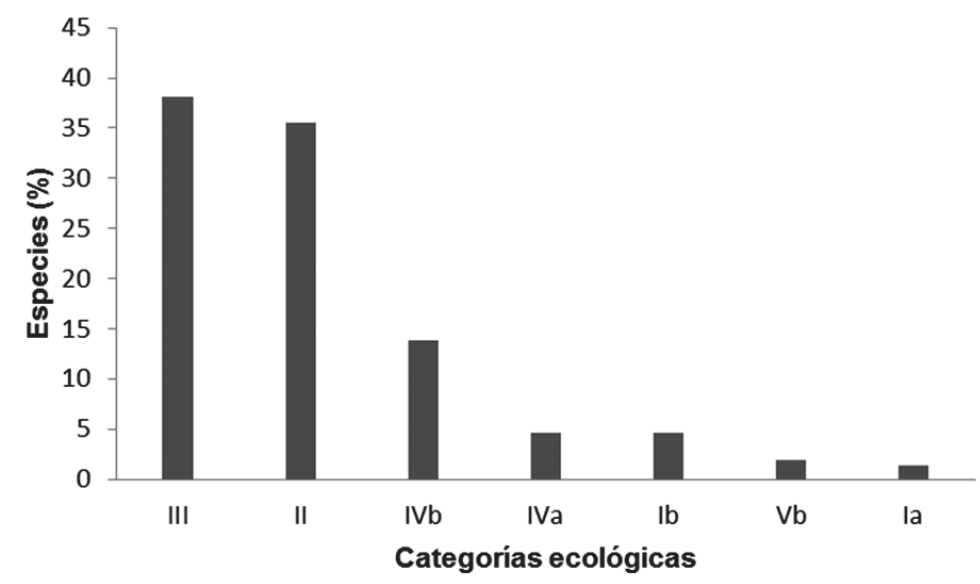

Figura 5. Porcentaje de especies por categoría ecológica en los 13 humedales de tierras bajas (<1000 m s.n.m.) del bosque seco tropical del departamento del Tolima durante los años 2010-2015.

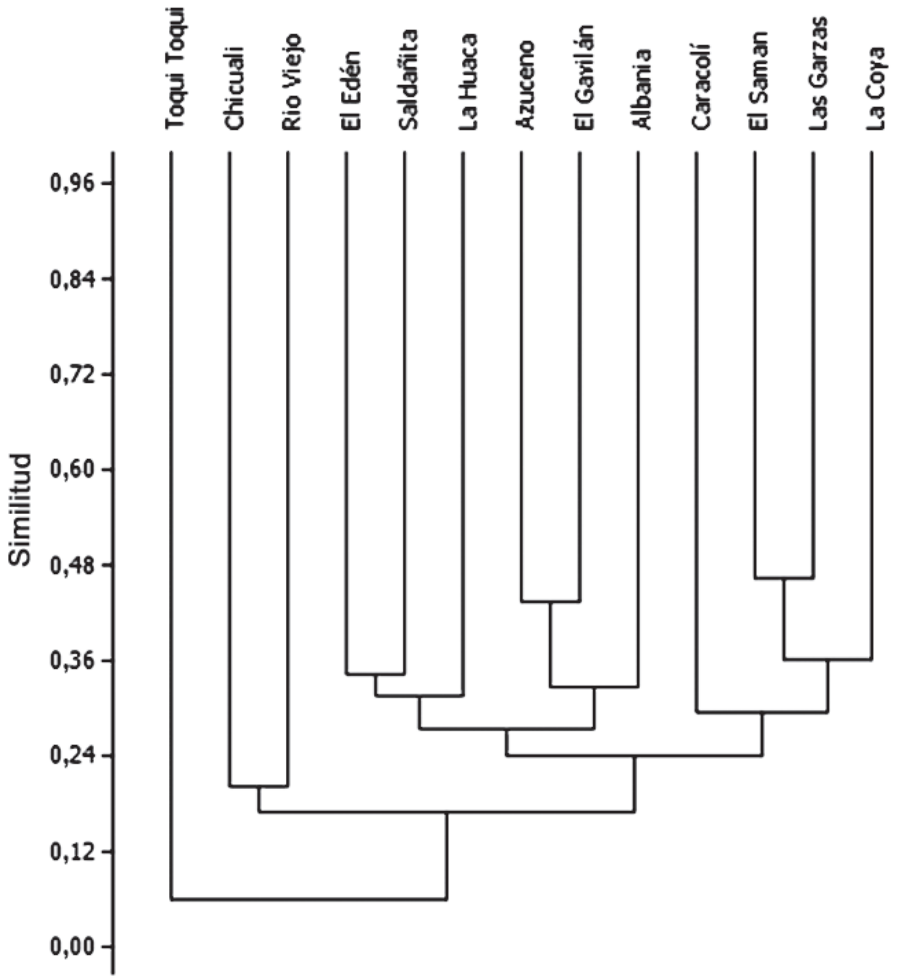

Figura 6. Análisis de similitud de Bray-Curtis para los 13 humedales de tierras bajas (<1000 m s.n.m.) del bosque seco tropical del departamento del Tolima durante los años 2010-2015. 


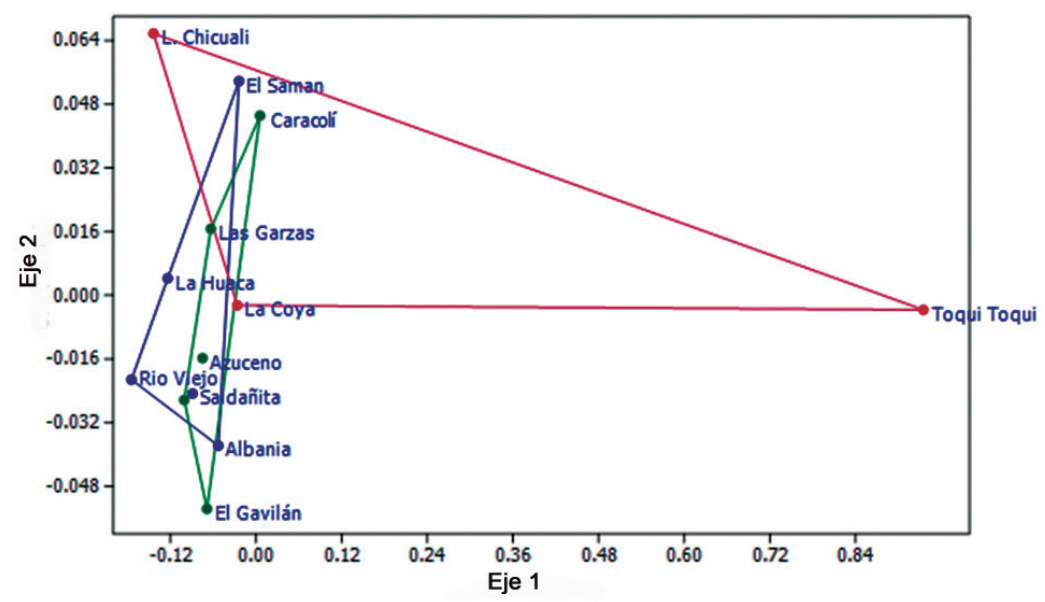

Figura 7. Diagrama de ordenación NMDS para los 13 humedales de tierras bajas (<1000 m s.n.m.) del bosque seco tropical del departamento del Tolima durante los años 2010-2015. Grupo I (línea verde), Grupo II (línea roja), Grupo III (línea azul).

\section{Discusión}

Se ha encontrado que la presencia de diferentes hábitats influye en la composición y abundancia de las aves (Cárdenas et al., 2003), en el caso de los humedales el área que abarca el espejo de agua, la profundidad y la presencia de diferentes hábitats a su alrededor determina las aves presentes en este tipo de ecosistemas (Elmberg et al., 1994; Green, 1996; Ntiamoa-Baidu et al., 1998). En referencia a los humedales que se estudiaron en este trabajo, los pastizales y los cultivos predominan en el entorno de todos los humedales, encontrándose además algunos relictos de bosque. Según Leveau y Leveau (2004) la cercanía a un casco urbano puede ser determinante en la composición de la avifauna; sin embargo, este factor no tuvo incidencia en los humedales evaluados, ya que no hubo diferencia en la composición de la avifauna entre humedales urbanos y no urbanos.

El análisis de las especies de acuerdo con la categoría ecológica estuvo acorde con lo anteriormente expuesto. Es decir, la presencia de pastizales, cultivos y matorrales alrededor de los humedales hizo que predominaran las especies de borde de bosque (categoría ecológica II) y áreas abiertas (categoría ecológica III). Enseguida estuvieron presentes las especies de aves acuáticas de la categoría ecológica IVb, esto pudo deberse a que los bordes de los humedales carecen de cobertura vegetal densa y las especies que hacen parte de esta categoría son aparentemente indiferentes a la presencia de árboles excepto para perchas (Stiles y Bohórquez, 2000). Además, la presencia de pocos relictos de bosque debido a la alta intervención a la cual se encuentra sometido el bosque seco tropical (Miles et al., 2006) y por ende los humedales evaluados, provocó que las especies de las categorías Ia y Ib fueran menos registradas, corroborando de este modo que la intervención del hábitat es un factor determinante en la diversidad de las aves (Cerezo et al., 2009; Ramírez-Albores, 2010).

Cabe resaltar que las especies de aves registradas en los humedales evaluados en el presente trabajo representa el $50 \%$ de la avifauna del bosque seco tropical del departamento del Tolima (LosadaPrado y Molina-Martínez, 2011), lo cual indica que los humedales y su hábitat circundante sustentan un número importante de aves representativas de esta zona de vida en esta parte del valle alto del río Magdalena, de las cuales se registraron 2 especies 
endémicas (Myiarchus apicalis y Euphonia concinna), 7 especies casi-endémicas y Phaethornis anthophilus como especie de interés (especies que tienen entre el 40-49 \% de su distribución en Colombia según Chaparro-Herrera et al., 2013); 7 especies migratorias (Naranjo et al., 2012); Psittacara wagleri como especie casi-amenazada (IUCN, 2017); Dendrocygna autumnalis dentro del apéndice III de la CITES y 20 especies dentro del apéndice II de la CITES (UNEP-WCMC, 2014). No obstante, es posible encontrar nuevos registros de aves para la región, por lo cual se recomienda seguir realizando monitoreos en estos 13 humedales y en otros que aún no han sido estudiados.

\section{Conclusiones}

Un significativo número de especies está asociado a los humedales de tierras bajas del Tolima. Entre ellas se destaca la presencia de especies de importancia para la conservación por su estatus endémico, migratorio, de interés o por estar en algún grado de amenaza (IUCN, CITES). Por último, la información obtenida en el presente estudio evidencia un alto registro de las especies esperadas, lo cual indica que se logró un buen muestreo; sin embargo, es posible seguir obteniendo nuevos registros para los humedales de zonas bajas del departamento del Tolima.

\section{Agradecimientos}

Este proyecto fue financiado por Cortolima en convenio con la Universidad del Tolima. Agradecemos a S. Espinosa por su ayuda en la elaboración del mapa y a J. García por aportar algunas de las fotos de las especies de aves registradas.

\section{Referencias}

Álvarez-León, R. (2009). Notas sobre la avifauna del humedal Santa María del Lago, cordillera Oriental de los Andes Colombianos. Revista Luna Azul, 28, 24-39.
Blanco, D. E. (1999). Los humedales como hábitat de aves acuáticas. En Malvárez, A. I. (Ed.). Tópicos sobre humedales subtropicales y templados de Sudamérica. Pp. 208-217. Montevideo: ORCYTUNESCO.

Brown, M. y Dinsmore, J. (1986). Implications of marsh size and isolation for marsh bird management. Journal of Wildlife Management, 50, 392-397.

Cárdenas, G., Harvey, C. A., Ibrahim, M. y Finegan, B. (2003). Diversidad y riqueza de aves en diferentes hábitats en un paisaje fragmentado en Cañas, Costa Rica. Agroforestería en las Américas, 10, 78-85.

Castillo, F. L. y Puyana, J. (2004). Colombia (Informe anual 2004). En Lopez-Lanus, B. y Blanco, D. E. (Eds). El Censo Neotropical de Aves Acuáticas 2004. Una herramienta para la conservación. Pp. 5363. Buenos Aires, Wetlands International.

Cerezo A., Robbins, C. S. y Dowell, B. (2009). Uso de hábitats modificados por aves dependientes de bosque tropical en la región caribeña de Guatemala. Revista de Biología Tropical, 57(1-2), 401-419.

Chaparro-Herrera S., Echeverry-Galvis, M. A., Córdoba-Córdoba, S. y Sua-Becerra, A. (2013). Listado actualizado de las aves endémicas y casiendémicas de Colombia. Biota Colombiana, 14(2), 235-272.

Colwell, R. K. (2013). Estimates: Statistical estimation of species richness and shared species from samples. Version 9.

Craig, R. J. y Beal, K. G. (1992). The influence of habitat variables on marsh bird communities of the Connecticut River Estuary. Wilson Bulletin, 104, 295-311.

Cruz, H. F., Moreno, M., Gómez, C. y Molina, Y. (2010). Aves. En Reinoso, G., Villa, F. A., Losada, S., García, J. E. y Vejarano, M. A. Biodiversidad faunistica de los humedales del departamentos del Tolima (Informe técnico). Pp. 306-413. Ibagué: Cortolima- Universidad del Tolima. 
Delany, S., Reyes, C., Hubert, E., Phil, S., Rees, E., Haanstra, L. y Van Strien, A. (1999). Results from the International Waterbird Census in the Western Palearctic and Southwest Asia 1995 and 1996. Wageningen: Wetlands International Publication.

Elmberg, J., Nummi, P., Pöysä, H. y Sjöberg, K. (1994). Relationships between species number, lake size and resource diversity in assemblages of breeding waterflowl. Journal of Biogeography, $21,75-84$.

Estela, F. A. y Lopez-Victoria, M. (2005). Aves de la parte baja del río Sinú, Caribe Colombiano; Inventario y ampliaciones de distribución. Boletín de Investigaciones Marinas y Costeras, 34, 7-42.

Green, A. J. (1996). Analyses of globally threatened Anatidae in relation to threats, distribution, migration patterns and habitat use. Conservation Biology, 10, 1435-1445.

Gutiérrez, F. P., Morales-B., D. y Lasso, C. A. (2014). Humedales interiores de Colombia: principales investigaciones, tipologías y propuestas de clasificación. En Lasso, C. A., Gutiérrez, F. de P. y Morales-B, D. (Eds). X. Humedales interiores de Colombia: identificación, caracterización y establecimiento de limites según criterios biológicos y ecológicos. Serie Editorial Recursos Hidrobiológicos y Pesqueros Continentales de Colombia. Pp. 67-87. Bogotá, D.C.: Instituto de Investigación de Recursos Biológicos Alexander von Humboldt.

Hammer O., Harper, D. A. T. y Ryan, P. D. (2001). PAST: Paleontological Statistic software. Palaeontología Electrónica.

IUCN. (2017). The IUCN Red List of Threatened Species. Version 2017-2. Recuperado de www. iucnredlist.org.

Johnston-González R., Castillo, F. L. y Murillo, J. (2006). Conocimiento y conservación de aves playeras en Colombia. Cali: Asociación Calidris. 29 pp.

Lara, C. E. (2011). Riqueza y composición de las aves del humedal "Ciénaga Colombia". Revista de Ciencias, 15, 201-207.
Lasso, C. A., Gutiérrez, F. de P. y Morales-B, D. (2014). X. Humedales interiores de Colombia: identificación, caracterización y establecimiento de límites según criterios biológicos y ecológicos. Serie Editorial Recursos Hidrobiológicos y Pesqueros Continentales de Colombia. Bogotá, D.C.: Instituto de Investigación de Recursos Biológicos Alexander von Humboldt. 349 pp.

Leveau, L. M. y Leveau, C. M. (2004). Comunidades de aves en un gradiente urbano de la ciudad de Mar del Plata, Argentina. Hornero, 19 (1), 13-21.

Losada-Prado, S. y Pacheco-Vargas, G. (2015a). Aves. En Reinoso, G. F. Villa, A., Losada-Prado, S. y Forero, A. M. Plan de manejo humedal Albania. Pp. 108-130.Ibagué: Cortolima-Universidad del Tolima.

Losada-Prado, S. y Pacheco-Vargas, G. (2015b). Aves. En Reinoso, G. F. Villa, A., Losada-Prado, S. y Forero, A. M. Plan de manejo humedal Azuceno. Pp. 124-154. Ibagué: Cortolima-Universidad del Tolima.

Losada-Prado, S. y Pacheco-Vargas, G. (2015c). Aves. En Reinoso, G. F. Villa, A., Losada-Prado, S. y Forero, A. M. Plan de manejo humedal El Saman. Pp. 100-122. Ibagué: Cortolima-Universidad del Tolima.

Losada-Prado, S. y Pacheco-Vargas, G. (2015d). Aves. En Reinoso, G. F., Villa, A., Losada-Prado, S. y Forero, A. M. Plan de manejo humedal La Huaca. Pp. 119-155. Ibagué: Cortolima-Universidad del Tolima.

Losada-Prado, S. y Pacheco-Vargas, G. (2015e). Aves. En Reinoso, G. F., Villa, A., LosadaPrado, S. y Forero, A. M. Plan de manejo humedal Las Garzas. Pp. 120-148. Ibagué: CortolimaUniversidad del Tolima.

Losada-Prado, S. y Molina-Martínez Y. G. (2011). Avifauna del bosque seco tropical en el departamento del Tolima (Colombia): Análisis de la comunidad. Caldasia, 33, 271-94.

Losada-Prado, S., Sánchez, N., Galeano, C. y Vega, N. (2016a). Aves: humedal Chicoali, San Luis. (Informe técnico). Ibagué: CortolimaUniversidad del Tolima. 
Losada-Prado, S., Sánchez, N., Galeano C. y Vega, N. (2016b). Aves: Humedal El Gavilán, Carmen de Apicalá (Informe técnico). Ibagué: CortolimaUniversidad del Tolima.

Losada-Prado, S., Sánchez, N., Galeano, C. y Vega, N. (2016c). Aves: humedal Caracolí, Saldaña (Informe técnico). Ibagué: CortolimaUniversidad del Tolima.

Miles, L., Newton, A. C., DeFries, R. S., Ravilious, C., May, I., Blyth, S., Kapos, V. y Gordon, J. E. (2006). A global overview of the conservation status of tropical dry forests. Journal of Biogeography, 33, 491-505.

Naranjo, L. G., Andrade, G. y Ponce de León, E. (1998). Hacia la conservación de los humedales de Colombia: Bases científicas y técnicas para una politica nacional de humedales. Boletín No. 9. Bogotá, D.C. Colombia: Instituto de Investigación de Recursos Biológicos Alexander von Humboldt.

Naranjo, L. G., Amaya, J. D., Eusse-González, D. y Cifuentes-Sarmiento, Y. (2012). Guía de las especies migratorias de la biodiversidad en Colombia Aves Vol. 1. Bogotá, D.C.: Ministerio de Ambiente y Desarrollo Sostenible / WWF Colombia. 708 pp.

Ntiamoa-Baidu, Y., Piersma, T., Wiersma, P., Pool, M., Battley, P. y Gordon, C. (1998). Water depth selection, daily feeding routines and diets of waterbirds in coastal lagoons in Ghana. Ibis, 140, 89-103.

Parra, J. L. (2014).Uso de la biota acuática en la identificación, caracterización y establecimiento de límites en humedales interiores: Aves. En Lasso, C. A., Gutiérrez, F. de P. y Morales-B., D. (Eds). X. Humedales interiores de Colombia: identificación, caracterización y establecimiento de límites según criterios biológicos y ecológicos. Serie Editorial Recursos Hidrobiológicos y Pesqueros Continentales de Colombia. Pp. 150-155. Bogotá, D.C.: Instituto de Investigación de Recursos Biológicos Alexander von Humboldt.

Pizano, C y García, H. (2014). El bosque seco tropical en Colombia. Bogotá, D.C.: Instituto de
Investigación de Recursos Biológicos Alexander von Humboldt. 349 pp.

PRIMER-E, L. (2006). PRIMER 6. Plymouth: PRIMER-E Ltd

Ramírez-Albores, J. E. (2010). Diversidad de aves de hábitats naturales y modificados en un paisaje de la Depresión Central de Chiapas, México. Revista de Biología Tropical, 58 (1), 511-528.

Remsen, J. V., Areta, J. I., Cadena, C. D., Claramunt, S., Jaramillo, A., Pacheco, J. F., Pérez-Emán, J., Robbins, M. B., Stiles, F. G., Stotz, D. F. y Zimmer, K. J. (2017). A classification of the bird species of South America. American Ornithologists' Union. Recuperado de http://www.museum. lsu.edu/ Remsen/SACCBaseline.html.

Safran, R. J., Isola, C. R., Colwell, M. A. y Williams, O. E. (1997). Benthic invertebrates at foraging locations of nine waterbird species in managed wetlands of the northern San Joaquin Valley, California. Wetlands, 17, 407-415.

Sánchez-Azofeifa, G. A., Quesada, M., Rodríguez, J. P., Nassar, J. M., Stoner, K. E., Castillo, A., Garvin, T., Zent, E. L., Calvo-Alvarado, J. C., Kalacska, M. E. R., Fajardo, L., Gamon, J. A., y Cuevas-Reyes, P. (2005). Research priorities for Neotropical dry forests. Biotropica, 37, 477-485.

Stiles, F. G. y Bohórquez, C. I. (2000). Evaluando el estado de la biodiversidad: el caso de la avifauna de la serranía de la Quinchas, Boyacá, Colombia. Caldasia, 22, 61-92.

UNEP-WCMC. (2014). Checklist of CITES species. Geneva, Suiza y Cambridge, Reino Unido: CITES Secretariat y UNEP-WCMC.

Velasquez, C. R. (1992). Managing artificial salipans as a waterbird hábitat: species' responses to water level manipulation. Colonial Waterbirds, 15, 14-55.

Weller, M. W. (1994). Seasonal Dynamics of Bird Assemblages in a Texas Estuarine Wetland (Dinámica Estacional de Agregaciones de Aves en un Estuario en Texas). Journal of Field Ornithology, 388-401. 
Anexo 1. Ubicación de los humedales de tierras bajas muestreados en el bosque seco tropical del departamento del Tolima.

Anexo 2. Taxonomía, categoría ecológica, estatus, grado de amenaza (IUCN) y CITES de las especies de aves registradas en los 13 humedales del bosque seco tropical del departamento del Tolima durante los años 2010-2015.

Disponible en línea: http:/ / revistas.humboldt.org.co/index.php/biota/rt/suppFiles/577/0

\section{Gustavo Pacheco-Vargas}

Universidad del Tolima,

Grupo de Investigación en Zoología

Tolima, Colombia

sfpacheco@ut.edu.co

Jessica Sánchez-Guzmán

Universidad del Tolima,

Grupo de Investigación en Zoología

Tolima, Colombia

jnsanchez@ut.edu.co

\section{Sergio Losada-Prado}

Universidad del Tolima,

Facultad de Ciencias,

Departamento de Biología

Tolima, Colombia

slosada@ut.edu.co
Caracterización de la comunidad de aves asociada a los humedales de zonas bajas del departamento del Tolima, Colombia

Citación del artículo: Pacheco-Vargas, G., Sánchez-Guzmán, J. y Losada-Prado, S. (2018). Caracterización de la comunidad de aves asociada a los humedales de zonas bajas del departamento del Tolima, Colombia. Biota Colombiana, 19(1), 190201. DOI: 10.21068/c2018.v19n01a12.

Recibido: 22 de agosto de 2017

Aprobado: 27 de marzo de 2018 